\title{
Endoscopic Resection of Functional Paraganglioma of the Pterygopalatine Fossa: A Case Report
}

\author{
Jae Hyuk Choi, MD, Eunkyu Lee, MD, and Sang Duk Hong, MD, PhD \\ Department of Otorhinolaryngology-Head and Neck Surgery, Samsung Medical Center, Sungkyunkwan University School of Medicine, \\ Seoul, Korea
}

Functional paraganglioma is a rare tumor that secretes the catecholamine which is able to cause secondary hypertension. Surgical resection is the only curative treatment modality for this tumor, which can correct the secondary hypertension. The pterygopalatine fossa is a small retromaxillary space which contains a neurovascular bundle that crosses the middle cranial fossa to the nasal cavity, nasal septum, and oral cavity. To our knowledge, two cases have been reported for functional paraganglioma involving the pterygopalatine fossa removed by surgery. We present a patient who had a catecholamine-secreting paraganglioma in the pterygopalatine fossa, which is the first case successfully resected with an endoscopic approach.

KEY WORDS: Functional paraganglioma · Pterygopalatine fossa $\cdot$ Endoscopic surgery.

\section{INTRODUCTION}

Paraganglioma is a vascular neoplasm that arises from paraganglionic cells. Except for pheochromocytoma, which occurs in the adrenal glands, $85 \%$ of paragangliomas occur in the abdomen, $12 \%$ in the thorax, and 3\% in the head and neck. ${ }^{1)}$ They are often diagnosed from an incidental finding on imaging or from symptoms related to mass effect, especially in head and neck paragangliomas that can present as cervical mass, headache, dysphagia, cranial nerve palsies, and visual loss. In cases of functional paraganglioma, catecholamine excess symptoms (e.g., heavy sweating, flushing, blurred vision, and headache) can occur. ${ }^{2)}$

Treatment options for paragangliomas include surgical resection, radiation therapy, radiotherapy, and chemotherapy. Traditionally, when the tumor is surgically resectable, surgery is the treatment of choice for paraganglioma. However, if the tumor is surgically unresectable for various reasons such as size and surgical morbidity, radiation ther- apy and radiotherapy could be considered for relieving symptoms. In the palliative setting, chemotherapy can be considered. ${ }^{3)}$

In the head and neck area, paragangliomas are rare and account for $0.6 \%$ of head and neck tumors. ${ }^{4)}$ Furthermore, functional paragangliomas comprise less than $1 \%$ of all head and neck paragangliomas. ${ }^{5)}$ Moreover, paragangliomas arising from the pterygopalatine fossa are extremely rare because most head and neck paragangliomas originate at the jugular bulb, vagus nerve, and carotid bifurcation. ${ }^{\text {) }}$ There are only two case reports of a functional paraganglioma arising from the pterygopalatine fossa removed by surgery, and to the best of our knowledge, there are no reported cases of endoscopic removal of a functional paraganglioma located in the pterygopalatine fossa in the English literature. ${ }^{78)}$

Herein, we report a case of a functional paraganglioma originating from the pterygopalatine fossa that was asymptomatic and incidentally discovered on computed tomography $(\mathrm{CT})$. The tumor was resected through an endoscop-

Received: November 26, 2019 / Revised: December 29, 2019 / Accepted: December 30, 2019

Address for correspondence: Sang Duk Hong, MD, PhD, Department of Otorhinolaryngology-Head and Neck Surgery, Samsung Medical Center, Sungkyunkwan University School of Medicine, 81 Irwon-ro, Gangnam-gu, Seoul 06351, Korea

Tel: +82-2-3410-3577, Fax:+82-2-3410-3879, E-mail: kkam97@gmail.com 
ic approach.

\section{CASE REPORT}

A 39-year-old asymptomatic woman, while undergoing a regular medical check-up, was found to have hypertension and an incidental mass in her right pterygopalatine fossa on a head and neck CT scan. An endoscopic biopsy, performed at another hospital, was conducted at the right maxillary posterior wall, where the mass was protruding, and revealed paraganglioma. An MRI scan was also obtained. Although the patient was offered radiation therapy to treat the paraganglioma, she visited our institution for a second opinion.

Upon presentation to our institution, she was taking valsartan $80 \mathrm{mg}$ for hypertension, and her average blood pressure, measured every time she visited the outpatient clinic, was 135/99. A CT scan demonstrated a $2.5 \mathrm{~cm}$-sized wellenhancing mass that was widening adjacent bony structures and eroding bones in the right pterygopalatine fossa. Enhancement was seen in the cavernous sinus superiorly and the sphenoid lateral recess posteriorly (Fig. 1). The tumor had low signal on a T1-weighted MRI image and a salt-and-
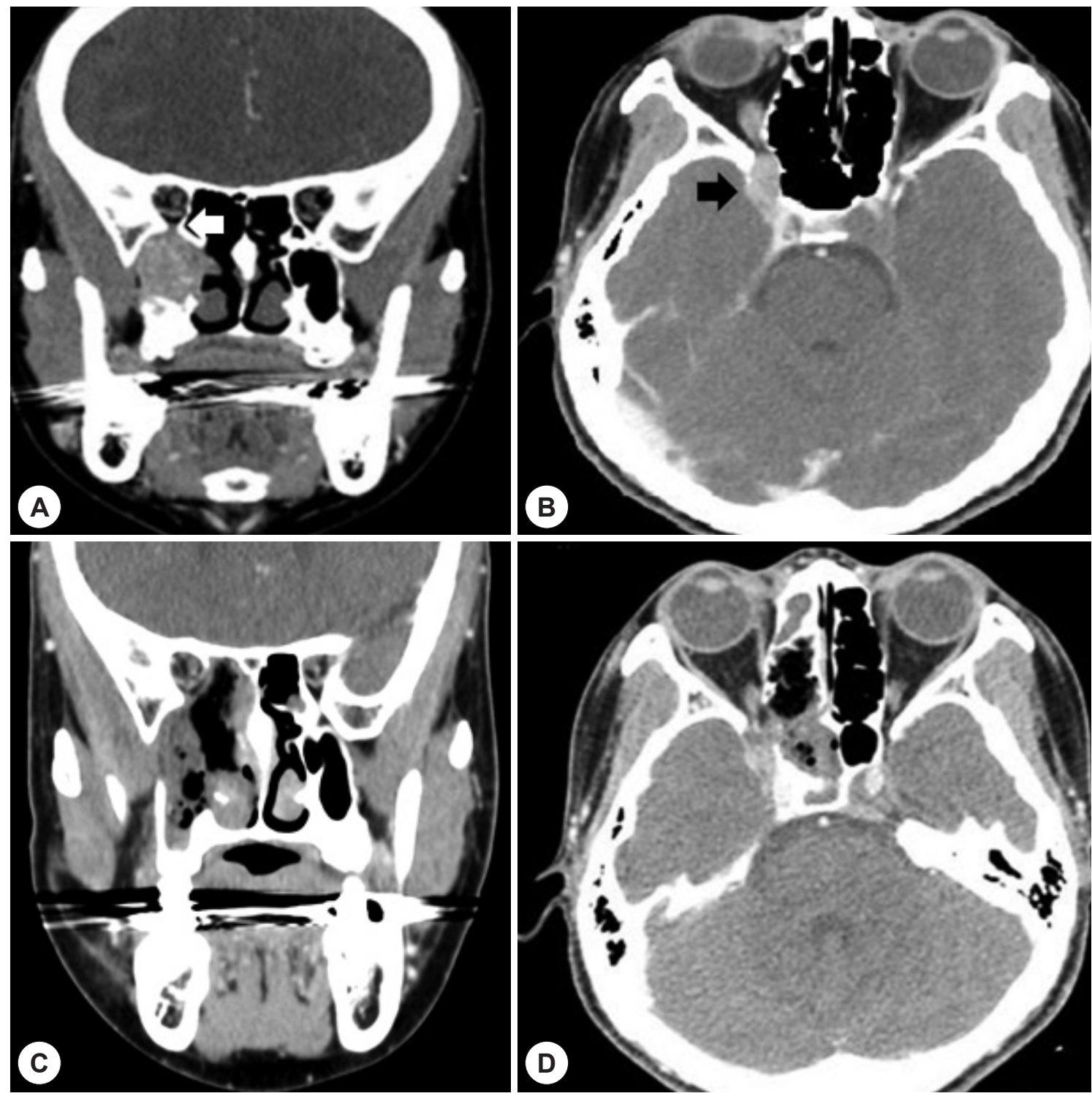

Fig. 1. A: Coronal view of contrast CT scan represents about 2-cm sized round enhancing mass in the right pterygopalatine fossa. The tumor causes widening of adjacent bony structure with extension to inferior orbital fissure (white arrow). B: Axial view of contrast CT represents that the tumor was adjacent to the cavernous sinus superiorly (black arrow) and the sphenoid lateral recess posteriorly. C, D: Immediate post-operative CT scan demonstrated no definite residual mass in pterygopalatine fossa. 
pepper pattern on the T2-weighted image, indicating that the mass was hypervascular (Fig. 2). The tumor didn't seem to invade the cavernous sinus on the MRI scan, unlike the CT scan. A urine test for catecholamines and metanephrines showed elevated normetanephrine and norepinephrine, suggesting the tumor was a functional paraganglioma (Table 1).

After reviewing all images and laboratory tests, we determined that surgical resection was possible with an endoscopic approach. The patient was counseled, and an endoscopic resection was planned to treat the patient.

One month before surgery, the patient required an additional medication, doxazosin, to control her blood pressure. Intravenous saline hydration was administered for 2 days before surgery to prevent a hypovolemic crisis after the operation.

Because paragangliomas have a high degree of vascularity, angiography and embolization were performed on the day before surgery to reduce operative blood loss. ${ }^{9)}$ After embolization of the right internal maxillary artery and the superior pharyngeal branch of the ascending pharyngeal artery, we confirmed the hypervascular tumor staining had decreased on angiography.

The patient underwent an endoscopic medial maxillectomy through a prelacrimal approach combined with the
Caldwell-Luc operation to approach the pterygopalatine fossa. The tumor was exposed after dissection of the maxillary posterior wall. Extracapsular dissection from muscle was performed by coblation with EVac, and the tumor was removed from dura of supeior orbital fissure using microdissector. The tumor margin, confirmed by the navigation system, extended laterally into the superior orbital fissure to the inferior orbital fissure, posteriorly into the lateral wall of the sphenoid sinus and superiorly into the cavernous sinus. The tumor measured $3.5 \times 3.2 \mathrm{~cm}$, and there was no vascular or capsular invasion. In the immunohistochemical analysis, Ki-67 was positive in 7\% of tumor cells. In pathology, nesting pattern of cells within a prominent vascular network is observed. There is no mitotic figures and no necrosis or chronic inflammation (Fig. 3)

Postoperatively, the patient experienced hypoesthesia in the right hard palate area. Her blood pressure was normal without any hypertensive medications (Table 1). On postoperative day one, $\mathrm{CT}$ revealed no definite residual mass (Fig. 1). Two weeks after surgery, a urine test for catecholamines and metanephrines revealed values in the normal range (Table 1). After two months, the patient has no complications and normal blood pressure without medication. A postoperative endoscopic exam demonstrated that the in-
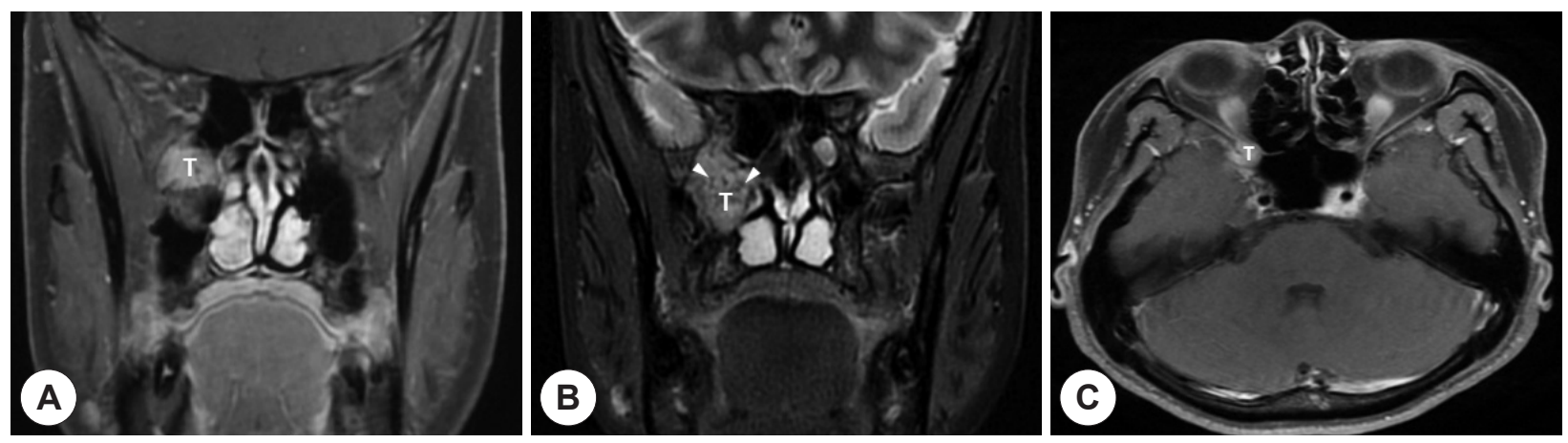

Fig. 2. A: In contrast-enhanced T1-weighted MRI scan, tumor (T) showed high signal intensity comparing to muscle. B: In T2-weighted MRI scan, the tumor (T) showed heterogeneous signal intensity and there are flow voids (white arrow heads) within the tumor caused by rapid vascular flow, which is a typical radiological finding in paraganglioma called "salt-and-pepper pattern." C: Axial contrast-enhanced Tl-weighted MRI scan. Though cavernous sinus invasion was suspected in CT scan, no definite cavernous sinus invasion was seen in MRI scan.

Table 1. Level of preoperative and postoperative urinary catecholamines and metanephrines

\begin{tabular}{lccc}
\hline Urinary catecholamines and metanephrines & Preoperative & Postoperative & Normal range \\
\hline VMA (mg/day) & 8.5 & 7.2 & $<6.8$ \\
Metanephrine (ug/day) & 53.2 & 38.9 & $<174.0$ \\
Normetanephrine (ug/day) & 1272.2 & 150.2 & $<471$ \\
Epinephrine (ug/day) & $<10$ & $<10$ & $0-20$ \\
Norepinephrine (ug/day) & 640 & 47.2 & $15-80$ \\
Dopamine (ug/day) & 248.6 & 151.0 & $65-400$ \\
\hline
\end{tabular}




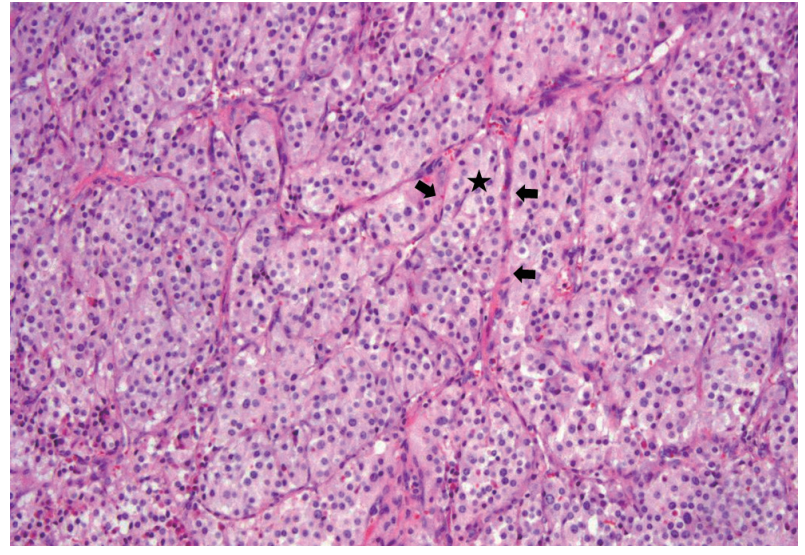

Fig. 3. Histopathologic examinations. The tumor shows typical Zellballen cluster formation (star) with prominent vascular network (black arrows) separating clusters of endocrine cells ( $\mathrm{H} \& \mathrm{E}, \times 100$ ). In Zellballen cluster, the endocrine cells with round nuclei containing abundant granular eosinophilic cytoplasm are observed.

ferior turbinate, maxillary posterior wall, and gingiva were well healed.

\section{DISCUSSION}

The pterygopalatine fossa is located in the deep face and is surrounded by the maxilla, pterygoid plate, ramus, and sphenoid bones. The maxillary division of the trigeminal nerve and vidian nerve pass through the pterygopalatine fossa, and pterygopalatine ganglion exist. Several tumors are known to occur in the pterygopalatine fossa, such as juvenile angiofibroma, nasopharyngeal carcinoma, and schwannoma. ${ }^{10)}$ In the present case, the pterygopalatine mass showed hypervascularity on the CT scan, so we doubted angiofibroma. But angiofibroma is extremely rare in a young female, so a biopsy was conducted and proved paraganglioma. It seems pterygopalatine fossa paragangliomas originate from paraganglionic tissue in the pterygopalatine fossa.

Treatment options for head and neck paragangliomas include observation, surgical resection, radiation therapy, and tumor embolization. If the tumor is surgically resectable, surgery is the treatment of choice. Radiation therapy and tumor embolization are options for symptom relief in cases of surgically unresectable head and neck paragangliomas. Because head and neck paragangliomas usually grow slowly, observation could be considered in elderly patients with medical comorbidities. ${ }^{4}$ In the present case, the tumor didn't appear to invade the cavernous sinus in the MRI scan, so we chose surgical treatment.

Traditionally, external surgical approaches, such as the
Fisch type $\mathrm{C}$ approach, infratemporal fossa approach and maxillary swing approach, were attempted to remove pterygopalatine fossa masses, including paragangliomas. In two cases of surgical removal of functional paraganglioma in pterygopalatine fossa, one used the the Fisch type C approach and the other was unknown. However, these approaches are too destructive and have poor visualization, and they have risks of hearing loss, neurovascular complications, sinus injuries, facial pain, and facial deformity. ${ }^{11)}$ Once the endoscopic approach to the pterygopalatine fossa was popularized, many tumors in pterygopalatine fossa have been endoscopically removed with different approaches, such as the transpterygoid approach, middle meatal transpalatine approach, and prelacrimal medial maxillectomy approach. ${ }^{12)}$

In the present case, we selected medial maxillectomy through a prelacrimal approach to obtain wide and clear visualization, prevent damage to the nasolacrimal duct and turbinate, and preserve nasal structures and function. Additionally, we combined this approach with the Caldwell-Luc approach to ease two-handed surgery and handle the instruments from various angles.

Although catecholamine secreting paraganglioma in the pterygopalatine fossa and superior orbital fissure is very rare and challenging, endoscopic transpterygoid approach could be a good surgical modality to cure the secondary hypertension caused by this rare tumor.

\section{REFERENCES}

1) Wasserman PG, Savargaonkar P. Paragangliomas: classification, pathology, and differential diagnosis. Otolaryngol Clin North Am 2001; 34(5):845-62, v-vi.

2) Young WF Jr. Paragangliomas: clinical overview. Ann N Y Acad Sci 2006;1073:21-9.

3) Martucci VL, Pacak K. Pheochromocytoma and paraganglioma: diagnosis, genetics, management, and treatment. Curr Probl Cancer 2014;38(1):7-41.

4) Boedeker CC, Ridder GJ, Schipper J. Paragangliomas of the head and neck: diagnosis and treatment. Fam Cancer 2005;4(1):55-9.

5) Williams MD. Paragangliomas of the head and neck: an overview from diagnosis to genetics. Head Neck Pathol 2017;11(3):278-87.

6) Hayward N, Cousins V. Head and neck paraganglioma: medical assessment, management, and literature update. J Otorhinolaryngol Hear Balance Med 2018;1:4.

7) Walker PJ, Fagan PA. Catecholamine-secreting paraganglioma of the pterygopalatine fossa: case report. Am J Otol 1993;14(3):306-8.

8) Otokida K, Ohira A, Kamimura A, Fujimori M, Kato M, Takahashi, et al. Cervical cathecolamine-secreting paraganglioma in the pterygopalatine fossa. Tohoku J Exp Med 1987;153(4):347-54.

9) White JB, Link MJ, Cloft HJ. Endovascular embolization of paragangliomas: a safe adjuvant to treatment. J Vasc Interv Neurol 2008; $1(2): 37-41$. 
10) Tashi S, Purohit BS, Becker M, Mundada P. The pterygopalatine fossa: imaging anatomy, communications, and pathology revisited. Insights Imaging 2016;7(4):589-99.

11) DelGaudio JM. Endoscopic transnasal approach to the pterygopalatine fossa. Arch Otolaryngol Head Neck Surg 2003;129(4):441-6.
12) Hofstetter CP, Singh A, Anand VK, Kacker A, Schwartz TH. The endoscopic, endonasal, transmaxillary transpterygoid approach to the pterygopalatine fossa, infratemporal fossa, petrous apex, and the Meckel cave. J Neurosurg 2010;113(5): 967-74. 